\title{
Hepatic duct confluence injury in blunt abdominal trauma - a diagnostic dilemma
}

\author{
Saurabh Garge, M.D., Kannan Lakshmi Narasimhan, M.D., \\ Shraddha Verma, M.D., Virender Sekhon, M.D.
}

Department of Pediatric Surgery, Pgimer, Chandigarh, India

\begin{abstract}
Isolated hepatic duct confluence injury due to trauma is unusual. Two cases of isolated bile duct injury are presented, which were diagnosed and managed successfully at our institution.
\end{abstract}

Key words: Hepatobiliary iminodiacetic acid scan, isolated hepatic duct injury.

\section{INTRODUCTION}

Extra-hepatic bile duct injury is rare, and injury at the hepatic duct confluence is even rarer. The majority of bile duct injuries in children are caused by blunt abdominal trauma and are associated with other hepatic and pancreaticobiliary injuries. In our cases, the injury was an isolated duct injury and was also in an unusual site. Duct injuries without any associated hepato- or pancreaticobiliary injuries, i.e., isolated common hepatic duct injuries, are rarer. The diagnosis poses a challenge and hence is often delayed. This report presents two cases of isolated hepatic duct confluence injury, with their successful management.

\section{CASE REPORT}

Case I- A four-year-old male child was brought with $\mathrm{H} / \mathrm{O}$ alleged blunt trauma to the abdomen from a car about one month before. The child had been admitted and managed conservatively at a peripheral hospital for moderate hemoperitoneum and splenic laceration of about $1.5 \times 1.5 \mathrm{~cm}$ in the upper splenic pole diagnosed by abdominal sonography. Three days after discharge, the child was brought to us with c/o abdominal pain, abdominal distension, multiple episodes

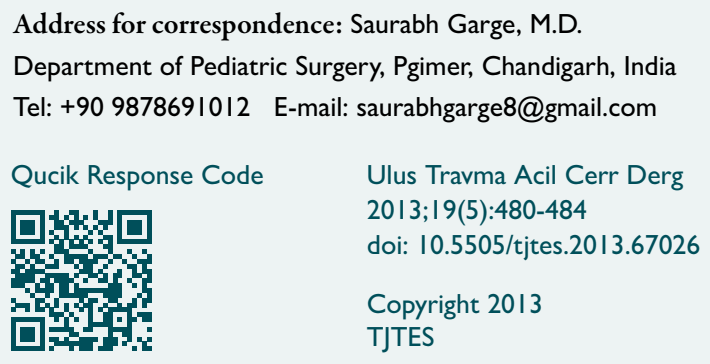

of non- bilious vomiting, and intermittent fever. Clinical examination revealed presence of tenderness in the epigastric region and left hypochondrium with presence of free fluid in the abdomen. The child was icteric. The biochemistry showed total serum bilirubin $5.5 \mathrm{~g} \%$ with direct fraction $2.5 \mathrm{~g} \%$. The serum alanine aminotransferase (ALT) and aspartate aminotransferase (AST) levels were found to be normal, with gross elevation of the alkaline phosphatase (ALP) levels (2836 IU) s/o cholestasis. A contrast-enhanced computed tomography (CECT) scan of the abdomen was done, which showed the presence of a linear laceration in the upper pole of the spleen of about $1.5 \times 2.0 \mathrm{~cm}$ s/o grade 2 splenic injury with moderate hemoperitoneum. All other viscera were essentially normal (Fig. I). The child was managed conservatively for two days, but due to persistent abdominal signs and jaundice, bile duct injury was suspected, and a HIDA (hepatobiliary iminodiacetic acid) scan was done. The HIDA scan showed normal hepatic uptake with absence of bilioenteric drainage and a bile leak in the peritoneum (Fig. 2).

The patient underwent a diagnostic laparoscopy, which revealed the presence of gross biliary fluid in the peritoneal cavity with bile staining at the porta hepatis. An intraoperative cholangiogram was performed, which showed the dye passing in the small bowel, showing patency of the distal tract and absence of any other ductal injuries (Fig. 3a). This was done because our case had a delayed presentation of bile leak, suggesting the presence of ischemic necrosis, which usually results in multiple sites of duct injury. $A 0.5 \times 0.8 \mathrm{~cm}$ perforation was found at the region of the confluence of the hepatic ducts. A $10 \mathrm{Fr}$ T-tube was placed through the tear and was brought outside the abdomen via the right lateral port, and the abdomen was closed with a subhepatic drain from the midline port. Postoperatively, the patient had an uneventful course. A repeat HIDA scan performed on postoperative day 


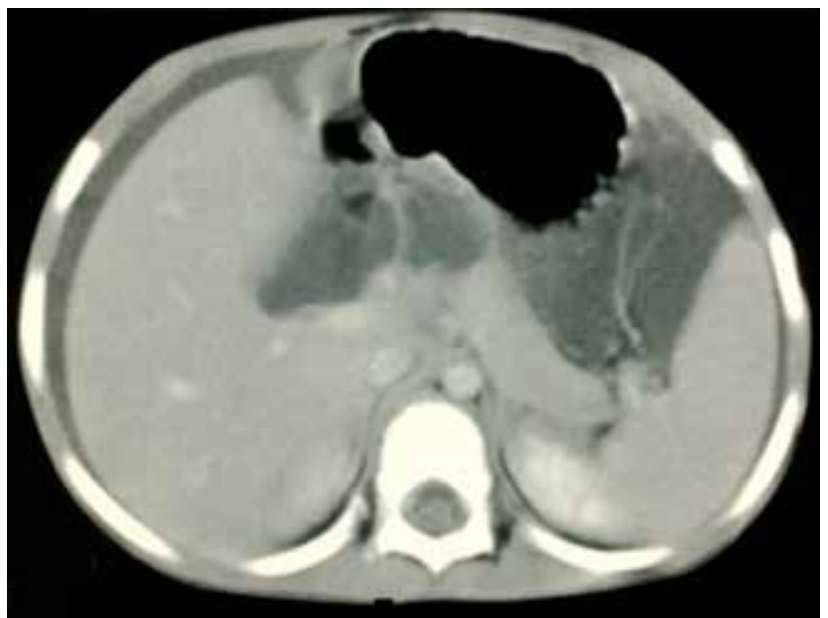

Figure 1. Computerized tomography scan showing splenic injury with hemoperitoneum.

6 showed no obstruction and normal drainage with no e/o bile leak (Fig. 3b). The T-tube was clamped subsequently. A T-tube cholangiogram performed on the 10th postoperative day showed a patent biliary tract with no leak. The T-tube was removed, and the patient was discharged on the IIth postoperative day with no specific complaints.

Case 2- A 10-year-old male child presented with alleged h/o blunt trauma to the abdomen due to being run over by the front wheel of a three-wheeler. The patient had no h/o unconsciousness, ear-nose-throat (ENT) bleed or respiratory distress. He had c/o a few episodes of vomiting but these were relieved with medications. The child was managed in a peripheral hospital where he had persistent hematuria on catheterization. An ultrasound performed showed presence of a renal injury. The patient was referred to our institution for further management. On arrival, the patient was found to be anemic and received 2 units of packed red cells. He had stable vitals and thus was conservatively managed. A CT scan was performed, which showed the presence of a linear hypodense region measuring $1.5 \mathrm{~cm} \times 0.7 \mathrm{~cm}$ in the midpolar region of the right renal cortex at its lateral margin. There was no evidence of any dye extravasation in the perinephric region. Adrenal hemorrhage on the right side was suspected. The other viscera including the liver were reported to be normal apart from some fluid collection in the lesser sac region (Fig. 4a). The patient had persistent abdominal distension while the hematuria subsequently subsided. The abdominal distension initially was gaseous but later became fluid with shifting dullness being elicitable. All the investigations in the patient were shown to be normal except for the bilirubin levels, which were persistently raised and showed an increasing trend. A HIDA scan was done to rule out presence of any bile leak. HIDA scan was s/o injury at the hepatic duct confluence (Fig. 4b). The patient underwent immediate exploration. Intraoperatively, there was confirmation of a defect in the region of the hepatic duct confluence of $0.5 \times 1.0 \mathrm{~cm}$. The peritonealized surface of the gallbladder was used for closing the tear. The patient had an uneventful postoperative course and was discharged on the 8th postoperative day with the cholecystostomy clamped. The cholecystostomy was removed on the 14th postoperative day.

\section{DISCUSSION}

Since the initial case report of bile duct rupture secondary to blunt trauma ${ }^{[1]}$ in 1799 , there have been only about 30 reports in the English-language literature from 1929 to 1995 involving injuries to the right and left hepatic ducts, either independently or at their bifurcation. ${ }^{[2,3]}$

The mechanisms of injury to the proximal bile duct are nu-

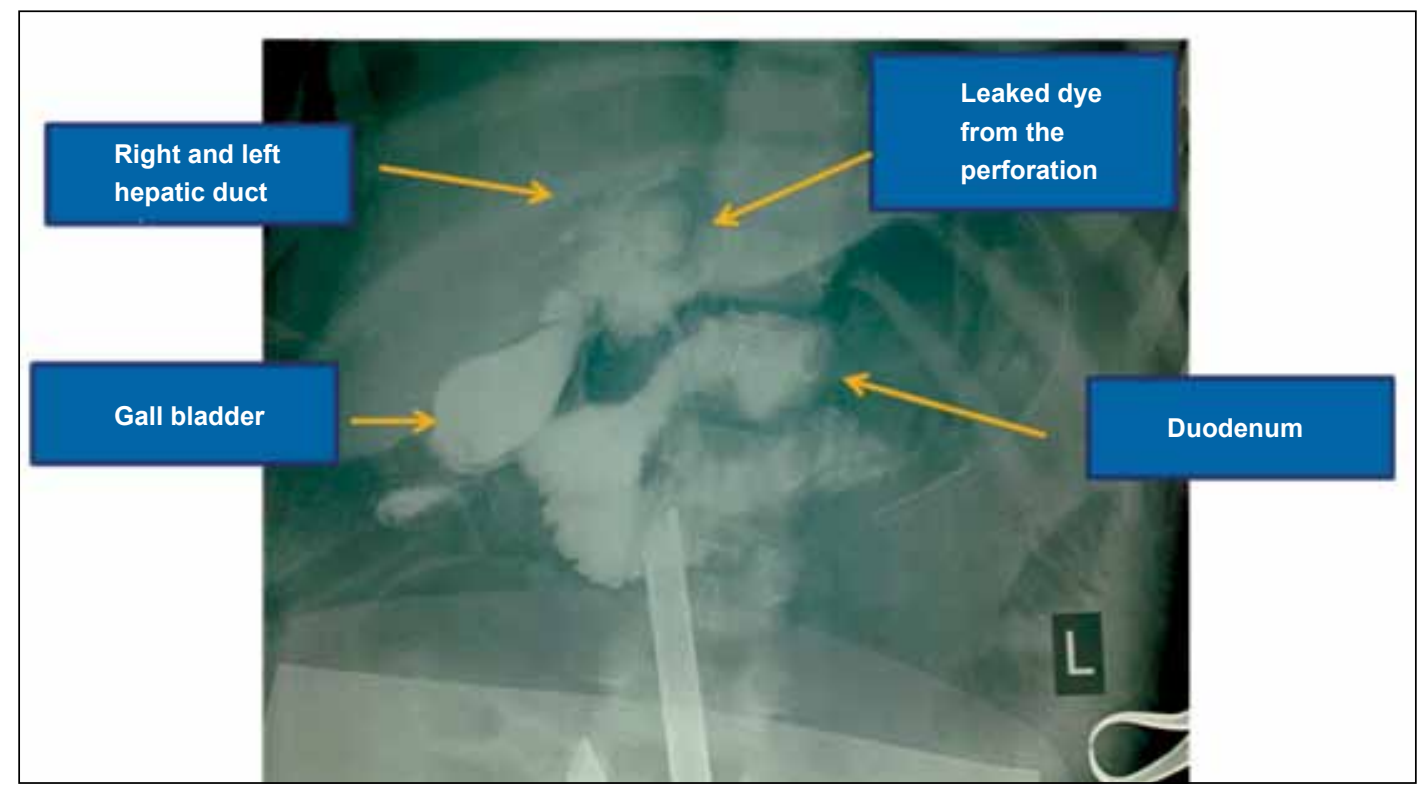

Figure 2. Intraoperative cholangiogram. 


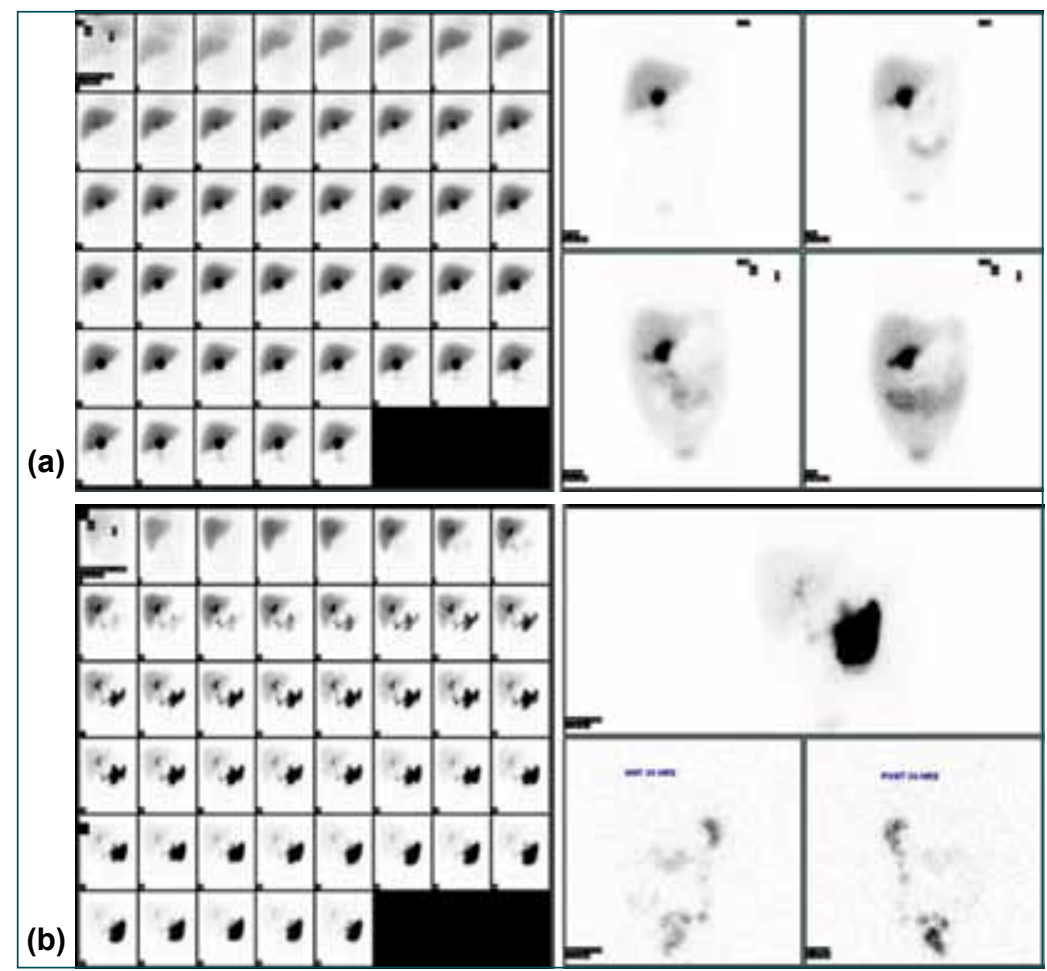

Figure 3. (a) Preoperative HIDA scan. (b) Postoperative HIDA scan.

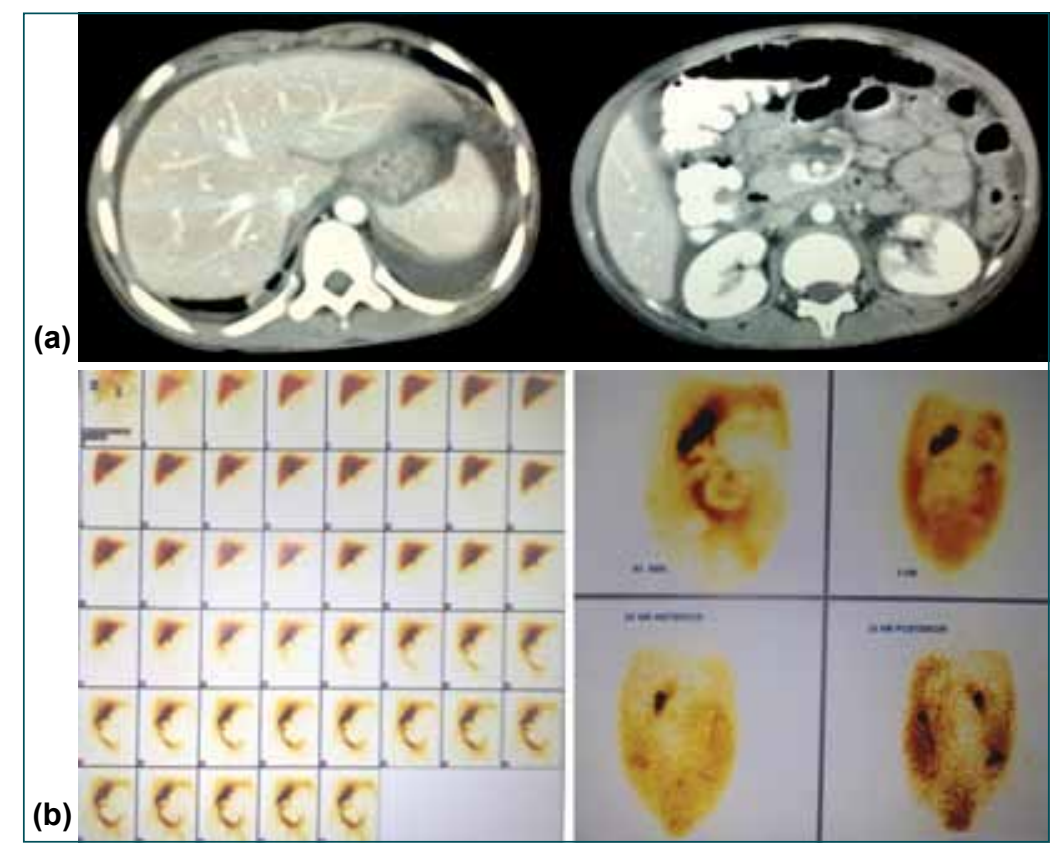

Figure 4. (a) Computerized tomography scan showing renal injury with collection in the lesser sac. (b) Preoperative HIDA scan.

merous and largely speculative, and include shearing forces applied to a distended bile duct at the area of maximum fixation. ${ }^{[3-8]}$ These areas of maximal fixation are $(I)$ the origin of the left hepatic duct, (2) the bifurcation of the hepatic ducts, and (3) the pancreaticoduodenal junction. Fletcher ${ }^{[9]}$ attributed three important factors to bile duct injury in blunt trauma: (I) a short cystic duct with rapid emptying of the gallbladder,
(2) direct force on the gallbladder causing its rapid emptying, and (3) shearing force applied to the distended gallbladder causing it to tear at its points of fixation, i.e., its point of exit from the liver or its point of entry into the pancreas. The fact that our second patient had a delayed bile leak, secondary to injury of the confluence of the hepatic ducts, suggests an ischemic necrosis of the duct. 
Bile duct injuries can often be missed unless an assiduous search is made in any suspicious injury. The clinical course of these injuries is insidious and often delayed, with an average time of 18 days, until the development of obvious abdominal signs and symptoms. ${ }^{[4-6]}$ Our cases were diagnosed 5 and 6 days' post-admission. Bile is sterile and can produce minimal peritoneal irritation, so the course of presentation can be chronic, and consists of mild peritoneal signs, jaundice, lowgrade fever, and weight loss. Accumulation of free fluid in the abdominal cavity with subsequent diagnostic puncture, or an abdominal drain draining bile-stained fluid, may draw attention to a possible injury of the biliary system. ${ }^{[10]}$ In the first case, the diagnosis was made after the 5 th day of admission to the hospital but two months after the blunt trauma. The diagnosis was made based on the persistently raised bilirubin level, presence of subtle abdominal signs, biliary paracentesis, and the HIDA scan showing presence of bile leak.

Preoperative diagnosis of biliary tree injuries is difficult. CT scans, HIDA scans, endoscopic retrograde pancreatography (ERCP), and intraoperative cholangiograms have been used to detect occult ductal injuries in high-risk patients in whom there is a high index of suspicion. CT and ultrasonography are useful for revealing peritoneal fluid collection or biliary dilatation. The CT can also help in diagnosing significant injury to the liver, especially segment II, III, or IV, or the presence of bile staining of the retroperitoneum. ${ }^{[2,3,5,8]}$ These associated liver injuries should raise the suspicion of the biliary injury and lead to a HIDA scan for the diagnosis of ductal injuries. In our case, no associated liver injury was sustained. HIDA scanning is the preferred screening tool for suspected bile duct injury. Delayed images should always be obtained if bile leakage is suspected, even if the study is normal at one hour. HIDA scanning can also be used for following the patient postoperatively. ${ }^{[I]}$

Endoscopic retrograde cholangiopancreatography (ERCP) has been used to detect extravasation of contrast medium from the injured site in selected patients with biliary injury. ${ }^{[2]}$

A variety of treatment options have been used for biliary tract injuries, with the choice of treatment being dictated by the type and degree of injury and the general condition of the patient. Small tears have been treated using any of the following options: ERCP and stenting, ${ }^{[13]}$ primary repair, ${ }^{[15]}$ and patch repair with vein, serosa or jejunal patch. ${ }^{[13,14]}$ In addition, T-tube decompression ${ }^{[15]}$ and entero-hepato-duodenal ligamentostomy ${ }^{[16]}$ have been used in difficult cases. Choledochoenterostomy and hepaticoenterostomy have been used for major injuries of the ducts, including complete transection. ${ }^{[10,13,14]}$ In our cases, we used T-tube repair of the duct perforation in the first case, while in the second case, the peritonealized surface of the gallbladder was used as a patch to cover the perforation.
In conclusion, a high index of suspicion and an awareness of vague abdominal signs are necessary for an early diagnosis. In our opinion, bilirubin elevation, bilious paracentesis, and hepatic fracture involving the porta hepatis as shown on CT should prompt appropriate investigation to exclude extrahepatic biliary injury. 99m-Tc-dimethyl acetanilide iminodiacetic acid (HIDA) scanning is the preferred screening tool for suspected bile duct injury. Delayed images should always be obtained if bile leakage is suspected, even if the study is normal at one hour. A normal HIDA scan in the face of continued symptoms requires further evaluation, mainly ERCP.

Conflict of interest: None declared.

\section{REFERENCES}

1. Wainwright T,'Letter'. Med Phys;362-4.

2. Michelassi F, Ranson JH. Bile duct disruption by blunt trauma. J Trauma 1985;25:454-7.

3. Dawson DL, Johansen $\mathrm{KH}$, Jurkovich GJ. Injuries to the portal triad. Am J Surg 1991;161:545-51.

4. Busuttil RW, Kitahama A, Cerise E, McFadden M, Lo R, Longmire WP Jr. Management of blunt and penetrating injuries to the porta hepatis. Ann Surg 1980;191:641-8.

5. Bade PG, Thomson SR, Hirshberg A, Robbs JV. Surgical options in traumatic injury to the extrahepatic biliary tract. Br J Surg 1989;76:2568.

6. Zollinger RM Jr, Keller RT, Hubay CA. Traumatic rupture of the right and left hepatic ducts. J Trauma 1972;12:563-9.

7. Muin A, Leong YP, Tay SK. Laceration of the common hepatic duct bifurcation by blunt abdominal trauma. Injury 1992;23:422-3.

8. Feliciano DV. Biliary injuries as a result of blunt and penetrating trauma. Surg Clin North Am 1994;74:897-912.

9. Fletcher WS. Nonpenetrating trauma to the gallbladder and extrahepatic bile ducts. Surg Clin North Am 1972;52:711-7.

10. Bourque MD, Spigland N, Bensoussan AL, Garel L, Blanchard H. Isolated complete transection of the common bile duct due to blunt trauma in a child, and review of the literature. J Pediatr Surg 1989;24:1068-70.

11. Bin Yahib S, Al Rabeeah A, Al Sammarrai A. An unusual bile duct injury in a child after blunt abdominal trauma. J Pediatr Surg 1999;34:1161-3.

12. Sugimoto K, Asari Y, Sakaguchi T, Owada T, Maekawa K. Endoscopic retrograde cholangiography in the nonsurgical management of blunt liver injury. J Trauma 1993;35:192-9.

13. Eid A, Almogy G, Pikarsky AJ, Binenbaum Y, Shiloni E, Rivkind A. Conservative treatment of a traumatic tear of the left hepatic duct: case report. J Trauma 1996;41:912-3.

14. Monk JS Jr, Church JS, Agarwal N. Repair of a traumatic noncircumferential hepatic bile duct defect using a vein patch: case report. J Trauma 1991;31:1555-7.

15. Ivatury RR, Rohman M, Nallathambi M, Rao PM, Gunduz Y, Stahl WM. The morbidity of injuries of the extra-hepatic biliary system. J Trauma 1985;25:967-73.

16. Bar-Maor JA, Shoshany G. Traumatic rupture of the choledochus treated temporarily by Roux-en-Y entero-hepato-duodenal ligamentostomy. J Pediatr Surg 1994;29:1578-9. 
OLGU SUNUMU - ÖZET

\section{Künt karın travmasında kompleks hepatik kanal yaralanması-tanısal ikilem}

Dr. Saurabh Garge, Dr. Kannan Lakshmi Narasimhan, Dr. Shraddha Verma, Dr. Virender Sekhon

Çocuk Cerrahisi Kliniği, Pgimer, Chandigarh, Hindistan

Travmaya bağlı izole ve kompleks hepatik kanal yaralanmaları seyrek görülmektedir. Merkezimizde başarılıyla tanı ve tedavisi yapılan iki izole safra kanalı yaralanması sunulmaktadır.

Anahtar sözcükler: Hepatobiliyer iminodiasetik asit tarama, izole hepatik kanal yaralanması.

Ulus Travma Acil Cerr Derg 2013;19(5):480-484 doi: 10.5505/tjtes.2013.67026 